\title{
Video Article \\ Non-invasive Assessments of Subjective and Objective Recovery Characteristics Following an Exhaustive Jump Protocol
}

\author{
Erich Hohenauer $^{1,2,3}$, Peter Clarys ${ }^{3}$, Jean-Pierre Baeyens ${ }^{2,3,4}$, Ron Clijsen ${ }^{1,2,3}$ \\ ${ }^{1}$ Department of Business Economics, Health and Social Care, University of Applied Sciences and Arts of Southern Switzerland \\ 2 University College Physiotherapy "Thim van der Laan" \\ ${ }^{3}$ Department of Movement and Sport Sciences, Vrije Universiteit Brussel \\ ${ }^{4}$ Faculty of Medicine and Health Sciences, University of Antwerp
}

Correspondence to: Erich Hohenauer at erich.hohenauer@supsi.ch

URL: https://www.jove.com/video/55612

DOI: doi: $10.3791 / 55612$

Keywords: Medicine, Issue 124, Cryotherapy, cold cuff, muscle damage, exhaustive jump protocol, recovery, humans

Date Published: 6/8/2017

Citation: Hohenauer, E., Clarys, P., Baeyens, J.P., Clijsen, R. Non-invasive Assessments of Subjective and Objective Recovery Characteristics Following an Exhaustive Jump Protocol. J. Vis. Exp. (124), e55612, doi:10.3791/55612 (2017).

\section{Abstract}

Fast recovery after strenuous exercise is important in sports and is often studied via cryotherapy applications. Cryotherapy has a significant vasoconstrictive effect, which seems to be the leading factor in its effectiveness. The resulting enhanced recovery can be measured by using both objective and subjective parameters. Two commonly measured subjective characteristics of recovery are delayed-onset muscle soreness (DOMS) and ratings of perceived exertion (RPE). Two important objective recovery characteristics are countermovement jump (CMJ) performance and peak power output (PPO). Here, we provide a detailed protocol to induce muscular exhaustion of the frontal thighs with a self-paced, $3 \times 30$ countermovement jump protocol (30-s rest between each set). This randomized controlled trial protocol explains how to perform local cryotherapy cuff application $\left(+8{ }^{\circ} \mathrm{C}\right.$ for $\left.20 \mathrm{~min}\right)$ and thermoneutral cuff application $\left(+32{ }^{\circ} \mathrm{C}\right.$ for $\left.20 \mathrm{~min}\right)$ on both thighs as two possible post-exercise recovery modalities. Finally, we provide a non-invasive protocol to measure the effects of these two recovery modalities on subjective (i.e., DOMS of both frontal thighs and RPE) and objective recovery (i.e., CMJ and PPO) characteristics 24,48 , and $72 \mathrm{~h}$ postapplication. The advantage of this method is that it provides a tool for researchers or coaches to induce muscular exhaustion, without using any expensive devices; to implement local cooling strategies; and to measure both subjective and objective recovery, without using invasive methods. Limitations of this protocol are that the $30 \mathrm{~s}$ rest period between sets is very short, and the cardiovascular demand is very high. Future studies may find the assessment of maximum voluntary contractions to be a more sensitive assessment of muscular exhaustion compared to CMJs.

\section{Video Link}

The video component of this article can be found at https://www.jove.com/video/55612/

\section{Introduction}

Cryotherapy is a frequently used treatment modality to enhance post-exercise recovery ${ }^{1,2}$. The vasoconstrictive response of the body after exposure to cold is one of the main mechanisms of reducing the inflammatory processes ${ }^{3,4}$. Frequently used cryotherapy procedures include cold packs ${ }^{5}{\text { or } \text { cuffs }^{6} \text {, cold air }}^{7,8}$, cold water immersion (CWI) ${ }^{9}$, whole-body cryotherapy ${ }^{10,11}$, cooling vests ${ }^{12}$, and internal cooling methods ${ }^{13,14}$. However, internal cooling procedures have demonstrated contradictory results ${ }^{15,16}$.

Controversial results on recovery have also been reported after external local cooling applications. One study reported that post-exercise cryotherapy did not enhance vertical jump performance (VJP) but decreased subjective ratings of perceived exertion (RPE) compared to active recovery procedures ${ }^{17}$. In contrast, another study demonstrated that cryotherapy had no influence on post-exercise subjective RPE ${ }^{5}$. A metaanalysis by Hohenauer et al. indicated that post-exercise cryotherapy might have a positive effect on subjective recovery characteristics, with no effects on inflammatory markers ${ }^{1}$.

The majority of studies that determine the effects of cryotherapy on recovery involve expensive devices to induce muscular exhaustion ${ }^{7,18,19}$ and invasive procedures to assess blood plasma markers or cytokines ${ }^{19,20,21}$. The aims of the present protocol are to induce similar muscular exhaustion without the use of any device and to provide a non-invasive method to determine the effects of local cryotherapy cuff application on subjective and objective recovery characteristics. The rationale behind this method is that it provides a tool for other researchers or coaches to induce muscular exhaustion, without using any expensive devices; to implement local cooling strategies based on this protocol; and to measure both subjective and objective recovery, without using invasive methods. This might help to assess subjective and objective recovery characteristics following an exhaustive jump protocol in a sport-realistic, non-invasive setting. 
Published studies show that validated, non-invasive methods for evaluating subjective recovery characteristics (i.e., delayed-onset muscle soreness (DOMS) $)^{22}$ and RPE) ${ }^{23}$ have been used with success. Countermovement jump (CMJ) performance ${ }^{17,24}$, with a high test-retest reliability ${ }^{25,26}$, can also be used as a non-invasive method to assess objective recovery characteristics.

\section{Protocol}

Ethics Statement: The Swiss Cantonal Ethical Committee of Zurich approved this protocol (KEK-ZH No. 2015-0113)

\section{Recruitment of Participants}

1. Recruit participants through social media and flyers.

2. Screen and include participants who are university students between 18 and 30 years of age. Ensure that they can perform endurance sports a minimum of two times (minimum total time: $2 \mathrm{~h}$ ) and a maximum of three times (maximum total time: $3 \mathrm{~h}$ ) per week, that they are free of current pain symptoms, and that they have had no injuries in the torso or lower extremities in the previous 12 months.

3. Screen and exclude participants if they are allergic to cold (including Raynaud's disease), suffer from cardiovascular diseases or any other disease, must take any medication, are pregnant, or have skeletal deviations.

\section{Familiarization with the Testing Protocol (Day 1)}

1. One week prior to the experiment, instruct the participants to perform a maximum of $5 \mathrm{CMJs}$ on a jump mat (see the Table of Materials), with a 1-min resting period between each jump ${ }^{12,27}$. Instruct them to start from an upright position and to place their hands on their hips to eliminate arm swing. Permit a self-determined range of motion and contraction time during the CMJs.

2. Instruct the participants to return to the laboratory one week after the familiarization session in a pain-free state and without having performed exhaustive exercise $48 \mathrm{~h}$ prior to the experiment.

NOTE: The pain-free and exhaustion-free state will be assessed as the baseline measurements.

\section{Baseline Measurements (Day 2)}

1. Measure the standing body-height, body-weight, and estimated lower-body fat percentage of the participants three times in a row and calculate the mean values ${ }^{28}$.

2. Have the participants assess their individual DOMS of both frontal thighs on a visual analog scale (VAS; $0-10 \mathrm{~cm})^{5}$ during a $3-\mathrm{s}$ lasting squat position ${ }^{29}\left(90^{\circ}\right.$ knee angle). Ask the participants: "How sore are your frontal thighs?" Record the number from the VAS in mm. NOTE: The far-left endpoint of the scale $(0 \mathrm{~cm})$ represents "no soreness," and the far-right endpoint of the scale $(10 \mathrm{~cm})$ represents "severe soreness."

3. Have the participants rate their individual perceived exertion in an upright standing position using a $6-20$ BORG scale ${ }^{30}$. Ask the participants: "What is your actual level of perceived exertion?" Record the verbally communicated number.

NOTE: The participants rate this verbally by telling the researcher one number between 6 (no perceived exertion) and 20 (maximum perceived exertion).

4. Have the participants perform a maximum of $3 \mathrm{CMJs}^{12,27}$ (as practiced during the familiarization session), with a 30 -s pause between the three jumps, on a jump mat.

NOTE: The jump heights from the CMJs are indirectly recorded as the time in flight by the jump mat system.

5. Use the highest jump and calculate the peak power output (PPO) according to the formula used in the study of Sayers et al. ${ }^{31}$. $\mathrm{PPO}=(60.7 \times$ jump height $[\mathrm{cm}])+(45.3 \times$ body weight $[\mathrm{kg}])-2,055$

NOTE: The subjective ratings and the objective assessments will be repeated directly after the recovery application $(0 \mathrm{~h})$ and 24,48 , and 72 $\mathrm{h}$ after the exhaustive jump protocol (see below).

6. Randomly assign the participants either to the cold group or to the thermoneutral group by drawing lots.

\section{Exhaustive Jump Protocol}

1. Directly after the randomization, instruct the participants to get ready to perform the exhaustive jump protocol, without any warm-up exercises.

2. Have two researchers visually inspect the quality of the jumps (maximum jump performance and touching the ground with the fingers after each jump), verbally correct, and encourage the participants during the jump protocol.

3. Have the participants perform a maximum of $3 \times 30 \mathrm{CMJs}$ or until exhaustion at their own speeds, with a $30 \mathrm{~s}$ pause between the sets. NOTE: Allow the participants to sit down and drink water during the pause.

\section{Recovery Application}

1. After the exhaustive jump protocol, have a blinded researcher apply either a cold cuff or a thermoneutral cuff (see the Table of Materials) to a participant's thighs.

1. Place the participant in supine position and apply both cuffs directly onto the skin of each thigh to ensure full contact, but using minimum pressure to avoid any compression effects.

NOTE: Optionally, refill the tank of a continuous programmable cooling and heating device with a ready-to-use, non-toxic lubrication mixture consisting of propylene glycol and demineralized water to the maximum level.

2. Start the device and apply either the cold modality $\left(+8{ }^{\circ} \mathrm{C}\right)$ or the thermoneutral modality $\left(+32{ }^{\circ} \mathrm{C}\right)$ for a duration of 20 min. 
NOTE: Instruct the participants not to report information about their temperature sensation.

3. Take off the thigh cuffs after the application and shut down the device.

\section{Non-invasive Recovery Assessment After $0 \mathrm{~h}$}

NOTE: The researcher performing all recovery assessments should not be informed if the participants received a cold- or a thermoneutral-cuff application.

1. Have the participants rate their individual DOMS and RPE, as described in steps 3.2 and 3.3 .

2. Have the participants perform a maximum of $3 \mathrm{CMJs}$ and calculate the PPO, as described in steps 3.4 and 3.5 .

3. Instruct the participants to maintain their daily habits but to refrain from alcohol, sport, and recreational training during the $72 \mathrm{~h}$ recovery period. Instruct the participants to return to the laboratory exactly 24,48 , and $72 \mathrm{~h}$ after the exhaustive jump protocol.

\section{Non-invasive Recovery Assessment After $24 \mathrm{~h}$ (Day 3), 48 h (Day 4), and $72 \mathrm{~h}$ (Day 5)}

1. Have the participants rate their individual DOMS and RPE, as described in steps 3.2 and 3.3.

2. Have the participants perform a maximum of 3 CMJs and calculate the PPO, as described in steps 3.4 and 3.5 . NOTE: The protocol ends after the $72 \mathrm{~h}$ follow-up period (Day 5).

\section{Representative Results}

The schematic representation of the test protocol is presented in Figure 1. This section illustrates that this protocol was successful at inducing muscular exhaustion and at monitoring subjective and objective recovery characteristics during a $72 \mathrm{~h}$ follow-up period, without using invasive techniques (Figure 2 and Figure 3).

Goodall et al. studied DOMS in a $90^{\circ}$ squat position after a muscle damage protocol ${ }^{29}$. Likewise, the present study also assessed DOMS in this position. As described in previously published articles, the DOMS elevated directly after the recovery application $(0 \mathrm{~h})$ and after $48 \mathrm{~h}$ in both groups (Figure 2A) ${ }^{29,32}$. However, in both groups, these values did not return to baseline values after a $72 \mathrm{~h}^{2}$ recovery period ${ }^{32}$.

This protocol also allows for the observation of changes in overall perceived exertion (Figure 2B). In the present study, a 6-20 BORG scale was used as in the study of Rowsell et al. ${ }^{24}$. In accordance to the study of Minett et al., the RPE was elevated in both groups directly after the recovery application ${ }^{12}$. However, these values returned to baseline after $24 \mathrm{~h}$ in the thermoneutral group and remained at this level for up to $72 \mathrm{~h}$. In the cold group, the RPE values increased again after $48 \mathrm{~h}$ and remained at this level for up to $72 \mathrm{~h}$.

Figure 3A and Figure 3B show that the exhaustive jump protocol induced a decrease in jump height (CMJ) and PPO in both conditions directly after the recovery application $(0 \mathrm{~h})^{12,33}$. Both the jump height $(\mathrm{CMJ})$ and PPO increased after $24 \mathrm{~h}$ and again decreased after 48 and $72 \mathrm{~h}$ in the cold and thermoneutral groups. These results are in line with previously published articles ${ }^{24,33,34}$.

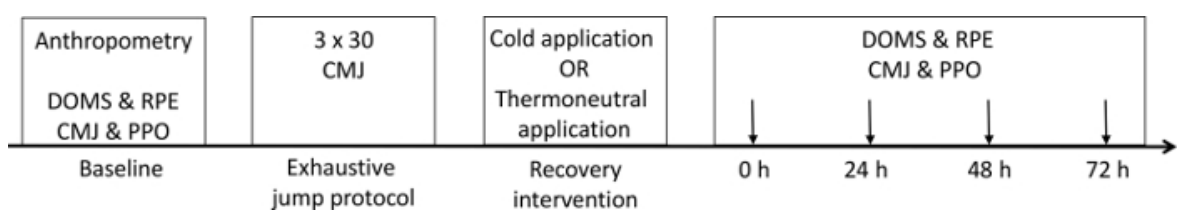

Figure 1: Schematic representation of the test protocol. Downward-pointing arrows denote the time points when recovery characteristics were measured. $\mathrm{DOMS}=$ delayed-onset muscle soreness, $\mathrm{RPE}=$ rated perceived exertion, $\mathrm{CMJ}=$ countermovement jump, $\mathrm{PPO}=$ peak power output. This figure has been modified from Hohenauer et al. $^{35}$. Please click here to view a larger version of this figure. 
A

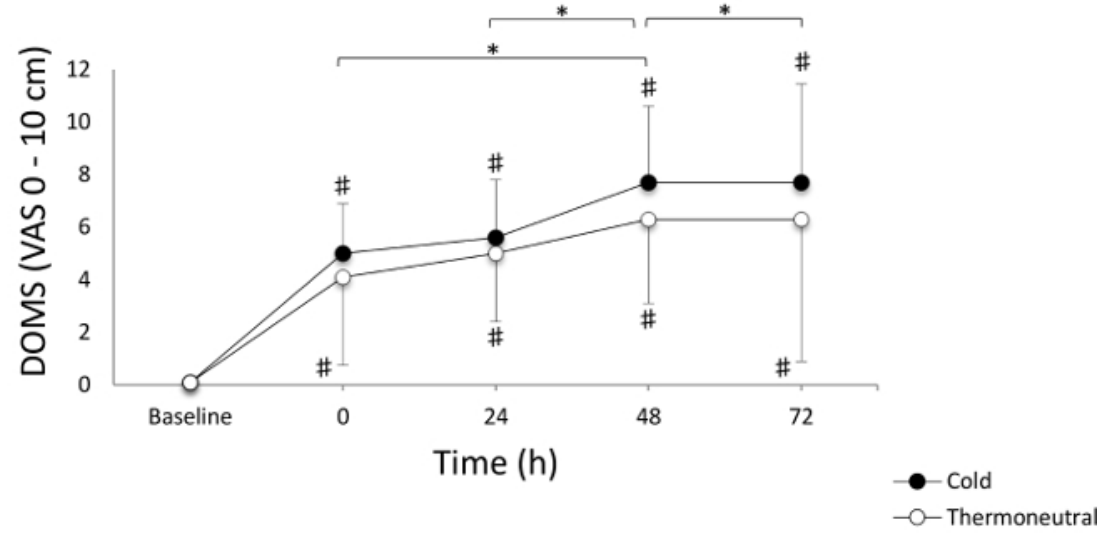

B

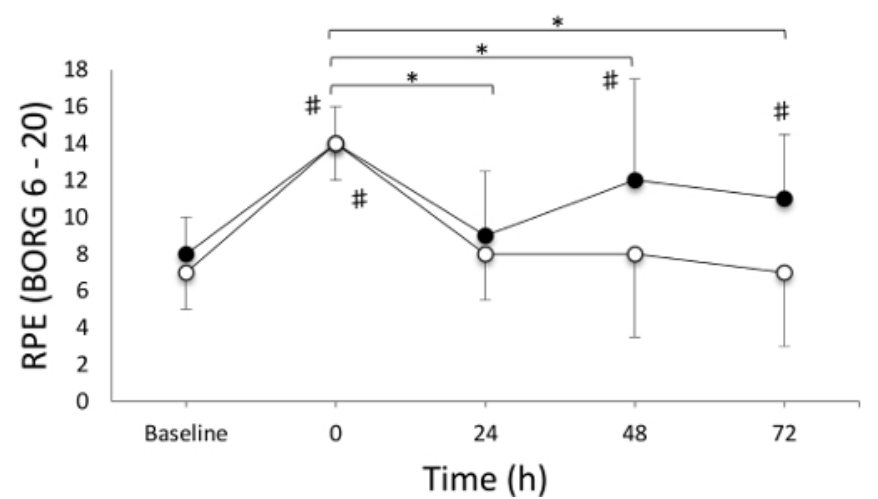

Figure 2: Change in DOMS and RPE over time. (A) Delayed-onset muscle soreness (DOMS; (median \pm interquartile ranges). A repeatedmeasures ANOVA revealed a time effect $(P=0.003)$ and a group effect $(P=0.03)$, but no group $x$ time interaction $(P=0.89)$. Post-hoc differences between the groups were $\mathrm{P}>0.05$ for all time points. (B) Rated perceived exertion (RPE; median \pm interquartile ranges). A repeatedmeasures ANOVA revealed a time effect $(P<0.001)$ and a group effect $(P=0.09)$, but no group $x$ time interaction $(P=0.29)$. Post-hoc differences between the groups were $P>0.05$ for all time points. ${ }^{*}$ indicates a significant time effect $(p<0.05)$. \# indicates significant differences from baseline values within groups $(p<0.05)$. This figure has been modified from Hohenauer et al. ${ }^{35}$. Please click here to view a larger version of this figure. 
A

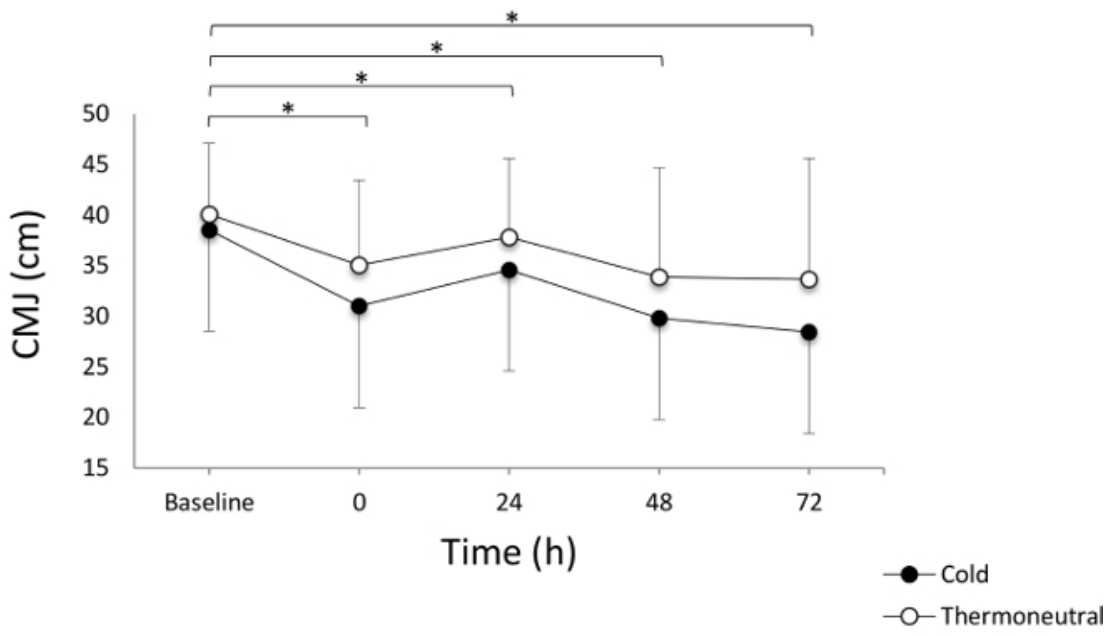

B

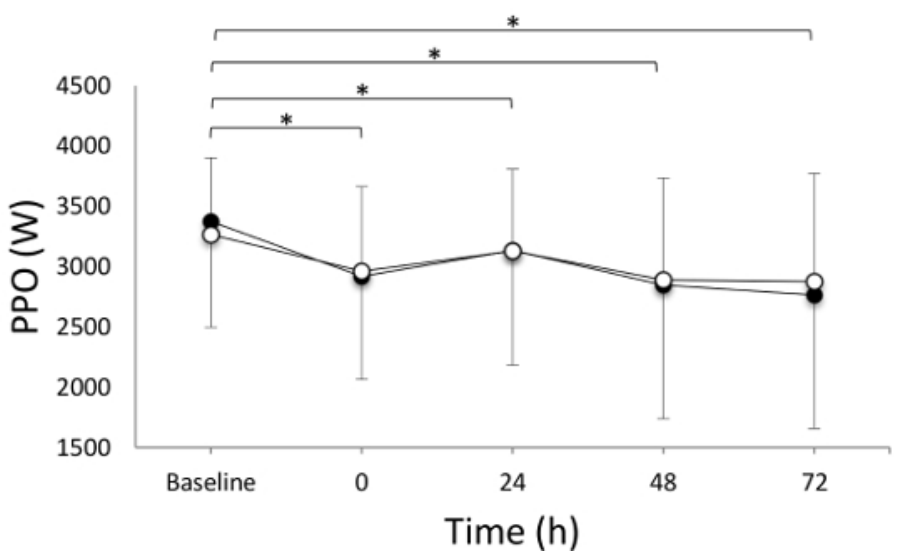

Figure 3: Change in CMJ and PPO over time. (A) Jump height during countermovement jump (CMJ; mean \pm SD). A repeated-measures ANOVA revealed a time effect $(P<0.001)$, but no group effect $(P=0.35)$ and time $x$ group interaction $(P=0.35)$. Post-hoc differences between the groups were $\mathrm{P}>0.05$ for all time points. (B) Peak power output (PPO; mean $\pm \mathrm{SD}$ ). A repeated-measures ANOVA revealed a time effect $(P$ $<0.001)$, but no group effect $(P=0.96)$ and time $x$ group interaction $(P=0.35)$. Post-hoc differences between groups were $P>0.05$ for all time points. * indicates a significant time effect $(\mathrm{P}<0.05)$. This figure has been modified from Hohenauer et al. ${ }^{35}$. Please click here to view a larger version of this figure.

\section{Discussion}

This protocol describes the execution of a standardized exhaustive jump protocol, a cryotherapy recovery modality, and a non-invasive assessment of recovery characteristics. The protocol of this randomized controlled trial provides several advantages compared to traditionally performed methods in the field of post-exercise recovery studies. Previous studies showed that jump protocols consisting of 100 jumps are a valid method to induce local muscle damage ${ }^{36,37}$. Ferreira-Junior et al. used a jump protocol consisting of five sets of 20 drop-jumps from a $0.6 \mathrm{~m}$ box, with $2 \mathrm{~min}$ rest intervals between the sets, to induce muscle damage of the frontal thighs $\mathrm{s}^{36}$. Whereas other traditional exhaustion protocols require expensive or mechanical devices ${ }^{38}$, the current protocol induced muscular exhaustion without the use of any mechanical device. Researchers do not need to buy or rent expensive devices to induce muscular exhaustion or damage to the frontal thighs.

The recovery interventions were applied using a continuous programmable cooling and heating device that can deliver cold or heat to a specific area of the body. Two thigh cuffs were applied around both thighs in supine position for 20 min. Although CWI is one of the most promising methods of enhancing recovery ${ }^{39}$, the transportation and the necessary amount of water are two challenging factors for the execution of this intervention. Furthermore, to guarantee the ideal water temperature, an additional person is needed to monitor and add ice.

An advantage of the present protocol is the use of an automated and portable cooling and heating device that ensures a constant temperature during the cuff application. Another advantage of the described protocol is the possibility of assessing subjective and objective recovery characteristics without taking blood samples from the participants. The subjective ratings, DOMS (VAS; 0-10 cm) and RPE (BORG; 6-20), used after an exhaustive protocol have been described in numerous published articles ${ }^{24,40,41,42}$. The assessment of CMJs, as presented in the present protocol, shows a high test-retest reliability (intra-class correlation coefficient (ICC) between 0.48 and 0.88 ) and validity ${ }^{25,26}$.

There are some critical steps within the protocol that may present potential sources of error. Two researchers must observe, verbally encourage, and correct the participants during the exhaustive CMJs $(3 \times 30)$. The objective monitoring of maximum CMJs may be ensured using accelerometers or linear transducers. Another critical step is the application of the two thigh cuffs. To avoid any compression effects ${ }^{43,44}$, the cuffs must be wrapped around each thigh with a minimum level of pressure. The application of the cuffs with a minimum of pressure might take a couple of practice runs to master. 
The present protocol has some limitations. The $30 \mathrm{~s}$ rest period between the sets of the exhaustive jump protocol is very short, and the cardiovascular impact is very high. Another limitation is the ready-to-use lubrication mixture for the recovery modality. It is important to take into account that the heat capacity of this lubrication mixture (i.e., propylene glycol and demineralized water) is slightly lower than that of normal water. The familiarization session of 5 jumps might be too small when the study population is not as physically active as described in the present protocol.

Finally, the assessment of CMJs presents the opportunity to assess objective recovery characteristics. Bishop et al. demonstrated a cheap and practicable method to assess jump height with the mobile-based app "My Jump," which has been shown to be a reliable method for measuring this variable ${ }^{45,46}$. However, Rowsell et al. indicated that no apparent reductions in CMJ height during a 5-day follow-up period could be observed after exhaustive soccer tournament matches ${ }^{24}$. Rupp et al. observed similar results after an exhaustive endurance test ${ }^{34}$. These results are in line with the results of the presents study, showing that the assessment of CMJ height might not be sensitive enough to measure the amount of muscular exhaustion.

In this protocol, the cold application temperature was set at $8^{\circ} \mathrm{C}$, whereas the temperature of the thermoneutral application was set at $32{ }^{\circ} \mathrm{C}$. It has been demonstrated that cold water temperatures are normally $\leq 20^{\circ} \mathrm{C}$ and that thermoneutral water temperatures have a range from $24{ }^{\circ} \mathrm{C}$ to $<36{ }^{\circ} \mathrm{C}^{47}$. It is important to consider that the amount of adipose tissue significantly affects the tissue cooling rate, with thicker skinfolds requiring longer application times ${ }^{48}$. Researchers should modify the cooling temperature and application duration according to their research populations

Future studies should consider that the assessment of the maximum voluntary contractions of the knee extensors might be a more sensitive assessment of objective recovery characteristics compared to $\mathrm{CMJs}^{49}$. For this protocol to be effective, it is crucial that the participants perform a familiarization session for the CMJs. Future studies using a different study population than that described here should increase the number of jumps to guarantee a familiarization effect. Additionally, future studies might increase the resting time between the exhaustive CMJs to guarantee maximum jump performance, which would then be unaffected by the high cardiovascular demand.

In conclusion, the current exhaustive jump protocol is an easy and practical way to induce muscular exhaustion of the frontal thighs without the use of any mechanical devices. By combining subjective (i.e., DOMS and RPE) and objective (i.e., CMJ and PPO) parameters, recovery can be investigated without taking any blood samples during a $72 \mathrm{~h}$ recovery period. The local post-exercise cryotherapy application can be carried out nearly anywhere and guarantees constant cooling temperatures.

\section{Disclosures}

None of the authors have competing or conflicting interests.

\section{Acknowledgements}

Thank you to the "Thim van der Laan" Foundation, Landquart, Switzerland, for the financial support. We also thank Thomas Konzett, University of Applied Sciences and Arts of Southern Switzerland, Landquart, Switzerland, and Ursula M. Küng, University College Physiotherapy, Landquart, Switzerland, for their assistance during the experiment, as well as Alexander Masselink, for his assistance with English.

\section{References}

1. Hohenauer, E., Taeymans, J., Baeyens, J. P., Clarys, P., \& Clijsen, R. The Effect of Post-Exercise Cryotherapy on Recovery Characteristics: A Systematic Review and Meta-Analysis. PLoS One. 10 (9), e0139028 (2015).

2. Costello, J. T. et al. Whole-body cryotherapy (extreme cold air exposure) for preventing and treating muscle soreness after exercise in adults. Cochrane Database Syst Rev. 9 CD010789 (2015).

3. White, G. E., \& Wells, G. D. Cold-water immersion and other forms of cryotherapy: physiological changes potentially affecting recovery from high-intensity exercise. Extrem Physiol Med. 2 (1), 26 (2013).

4. Mawhinney, C. et al. Influence of cold-water immersion on limb and cutaneous blood flow after exercise. Med Sci Sports Exerc. 45 (12), 2277-2285 (2013).

5. Tseng, C. Y. et al. Topical cooling (icing) delays recovery from eccentric exercise-induced muscle damage. J Strength Cond Res. 27 (5), 1354-1361 (2013).

6. Pointon, M., Duffield, R., Cannon, J., \& Marino, F. E. Cold application for neuromuscular recovery following intense lower-body exercise. Eur J Appl Physiol. 111 (12), 2977-2986 (2011).

7. Guilhem, G. et al. Effects of air-pulsed cryotherapy on neuromuscular recovery subsequent to exercise-induced muscle damage. Am J Sports Med. 41 (8), 1942-1951 (2013).

8. Leicht, A. S. et al. Influence of postexercise cooling techniques on heart rate variability in men. Exp Physiol. 94 (6), 695-703 (2009).

9. Ingram, J., Dawson, B., Goodman, C., Wallman, K., \& Beilby, J. Effect of water immersion methods on post-exercise recovery from simulated team sport exercise. J Sci Med Sport. 12 (3), 417-421 (2009).

10. Hausswirth, C. et al. Effects of whole-body cryotherapy vs. far-infrared vs. passive modalities on recovery from exercise-induced muscle damage in highly-trained runners. PLoS One. 6 (12), e27749 (2011).

11. Ferreira-Junior, J. B. et al. Effects of partial-body cryotherapy (- 110 degrees $C)$ on muscle recovery between high-intensity exercise bouts. Int J Sports Med. 35 (14), 1155-1160 (2014).

12. Minett, G. M., Duffield, R., Kellett, A., \& Portus, M. Effects of mixed-method cooling on recovery of medium-fast bowling performance in hot conditions on consecutive days. J Sports Sci. 30 (13), 1387-1396 (2012).

13. Stanley, J., Leveritt, M., \& Peake, J. M. Thermoregulatory responses to ice-slush beverage ingestion and exercise in the heat. Eur J Appl Physiol. 110 (6), 1163-1173 (2010).

14. Tran Trong, T., Riera, F., Rinaldi, K., Briki, W., \& Hue, O. Ingestion of a cold temperature/menthol beverage increases outdoor exercise performance in a hot, humid environment. PLoS One. 10 (4), e0123815 (2015). 
15. Siegel, R., Mate, J., Watson, G., Nosaka, K., \& Laursen, P. B. Pre-cooling with ice slurry ingestion leads to similar run times to exhaustion in the heat as cold water immersion. J Sports Sci. 30 (2), 155-165 (2012).

16. Hue, O. et al. The effect of time of day on cold water ingestion by high-level swimmers in a tropical climate. Int J Sports Physiol Perform. 8 (4), 442-451 (2013).

17. King, M., \& Duffield, R. The effects of recovery interventions on consecutive days of intermittent sprint exercise. J Strength Cond Res. 23 (6), 1795-1802 (2009).

18. Costello, J. T., Algar, L. A., \& Donnelly, A. E. Effects of whole-body cryotherapy (-110 degrees C) on proprioception and indices of muscle damage. Scand J Med Sci Sports. 22 (2), 190-198 (2012).

19. Sellwood, K. L., Brukner, P., Williams, D., Nicol, A., \& Hinman, R. Ice-water immersion and delayed-onset muscle soreness: a randomised controlled trial. Br J Sports Med. 41 (6), 392-397 (2007).

20. Ascensao, A., Leite, M., Rebelo, A. N., Magalhaes, S., \& Magalhaes, J. Effects of cold water immersion on the recovery of physical performance and muscle damage following a one-off soccer match. J Sports Sci. 29 (3), 217-225 (2011).

21. Yanagisawa, O. et al. The use of magnetic resonance imaging to evaluate the effects of cooling on skeletal muscle after strenuous exercise. Eur J Appl Physiol. 89 (1), 53-62 (2003).

22. Delextrat, A., Calleja-Gonzalez, J., Hippocrate, A., \& Clarke, N. D. Effects of sports massage and intermittent cold-water immersion on recovery from matches by basketball players. J Sports Sci. 31 (1), 11-19 (2013).

23. Stanley, J., Peake, J. M., \& Buchheit, M. Consecutive days of cold water immersion: effects on cycling performance and heart rate variability. Eur J Appl Physiol. 113 (2), 371-384 (2013).

24. Rowsell, G. J., Coutts, A. J., Reaburn, P., \& Hill-Haas, S. Effects of cold-water immersion on physical performance between successive matches in high-performance junior male soccer players. J Sports Sci. 27 (6), 565-573 (2009).

25. Markovic, G., Dizdar, D., Jukic, I., \& Cardinale, M. Reliability and factorial validity of squat and countermovement jump tests. J Strength Cond Res. 18 (3), 551-555 (2004).

26. Slinde, F., Suber, C., Suber, L., Edwen, C. E., \& Svantesson, U. Test-retest reliability of three different countermovement jumping tests. $J$ Strength Cond Res. 22 (2), 640-644 (2008).

27. Vieira, A. et al. Does whole-body cryotherapy improve vertical jump recovery following a high-intensity exercise bout? Open Access $J$ Sports Med. 6 49-54 (2015).

28. Kinanthropometry, T. I. S. F. T. A. o. International Standards for Anthropometric Assessment. National Library of Australia. (2001).

29. Goodall, S., \& Howatson, G. The effects of multiple cold water immersions on indices of muscle damage. J Sports Sci Med. 7 (2), 235-241 (2008).

30. Borg, G. A. Psychophysical bases of perceived exertion. Med Sci Sports Exerc. 14 (5), 377-381 (1982).

31. Sayers, S. P., Harackiewicz, D. V., Harman, E. A., Frykman, P. N., \& Rosenstein, M. T. Cross-validation of three jump power equations. Med Sci Sports Exerc. 31 (4), 572-577 (1999).

32. Howatson, G., Goodall, S., \& van Someren, K. A. The influence of cold water immersions on adaptation following a single bout of damaging exercise. Eur J Appl Physiol. 105 (4), 615-621 (2009)

33. Vaile, J., Halson, S., Gill, N., \& Dawson, B. Effect of hydrotherapy on the signs and symptoms of delayed onset muscle soreness. Eur J Appl Physiol. 102 (4), 447-455 (2008).

34. Rupp, K. A. et al. The effect of cold water immersion on 48-hour performance testing in collegiate soccer players. J Strength Cond Res. 26 (8), 2043-2050 (2012).

35. Hohenauer, E., Clarys, P., Baeyens, J. P., \& Clijsen, R. The effect of local cryotherapy on subjective and objective recovery characteristics following an exhaustive jump protocol. Open Access $J$ Sports Med. 7 89-97 (2016).

36. Ferreira-Junior, J. B. et al. One session of partial-body cryotherapy (-110 degrees C) improves muscle damage recovery. Scand J Med Sci Sports. 25 (5), e524-530 (2015).

37. Twist, C., \& Eston, R. The effects of exercise-induced muscle damage on maximal intensity intermittent exercise performance. Eur J Appl Physiol. 94 (5-6), 652-658 (2005).

38. Abaidia, A. E. et al. Recovery From Exercise-Induced Muscle Damage: Cold Water Immersion Versus Whole Body Cryotherapy. Int J Sports Physiol Perform. (2016).

39. Bleakley, C. et al. Cold-water immersion (cryotherapy) for preventing and treating muscle soreness after exercise. Cochrane Database Syst Rev. 2 CD008262 (2012).

40. Jakeman, J. R., Macrae, R., \& Eston, R. A single 10-min bout of cold-water immersion therapy after strenuous plyometric exercise has no beneficial effect on recovery from the symptoms of exercise-induced muscle damage. Ergonomics. 52 (4), $456-460$ (2009).

41. Crystal, N. J., Townson, D. H., Cook, S. B., \& LaRoche, D. P. Effect of cryotherapy on muscle recovery and inflammation following a bout of damaging exercise. Eur J Appl Physiol. 113 (10), 2577-2586 (2013).

42. Davies, R. C., Rowlands, A. V., \& Eston, R. G. Effect of exercise-induced muscle damage on ventilatory and perceived exertion responses to moderate and severe intensity cycle exercise. Eur J Appl Physiol. 107 (1), 11-19 (2009).

43. Davies, V., Thompson, K. G., \& Cooper, S. M. The effects of compression garments on recovery. J Strength Cond Res. 23 (6), 1786-1794 (2009).

44. Jakeman, J. R., Byrne, C., \& Eston, R. G. Lower limb compression garment improves recovery from exercise-induced muscle damage in young, active females. Eur J Appl Physiol. 109 (6), 1137-1144 (2010).

45. Bishop, C., Tarrant, J., Jarvis, P., \& Turner, A. Using The Split Squat To Potentiate Bilateral And Unilateral Jump Performance. J Strength Cond Res. (2016).

46. Balsalobre-Fernandez, C., Glaister, M., \& Lockey, R. A. The validity and reliability of an iPhone app for measuring vertical jump performance. J Sports Sci. 33 (15), 1574-1579 (2015).

47. Versey, N. G., Halson, S. L., \& Dawson, B. T. Water immersion recovery for athletes: effect on exercise performance and practical recommendations. Sports Med. 43 (11), 1101-1130 (2013).

48. Jutte, L. S., Merrick, M. A., Ingersoll, C. D., \& Edwards, J. E. The relationship between intramuscular temperature, skin temperature, and adipose thickness during cryotherapy and rewarming. Arch Phys Med Rehabil. 82 (6), 845-850 (2001).

49. Warren, G. L., Lowe, D. A., \& Armstrong, R. B. Measurement tools used in the study of eccentric contraction-induced injury. Sports Med. 27 (1), 43-59 (1999) 